\title{
Proper Time Delays Measured by Optical Streaking
}

\author{
Ulf Saalmann $\odot$ and Jan M. Rost \\ Max-Planck-Institut für Physik komplexer Systeme, Nöthnitzer Straße 38, 01187 Dresden, Germany
}

(Received 13 March 2020; accepted 20 August 2020; published 10 September 2020)

\begin{abstract}
In attosecond science it is assumed that Wigner-Smith time delays, known from scattering theory, are determined by measuring streaking shifts. Despite their wide use from atoms to solids this has never been proven. Analyzing the underlying process-energy absorption from the streaking light—we derive this relation. It reveals that only under specific conditions streaking shifts measure Wigner-Smith time delays. For the most relevant case, interactions containing long-range Coulomb tails, we show that finite streaking shifts, including relative shifts from two different orbitals, are misleading. We devise a new time-delay definition and describe a measurement technique that avoids the record of a complete streaking scan, as suggested by the relation between time delays and streaking shifts.
\end{abstract}

DOI: 10.1103/PhysRevLett.125.113202

Attosecond laser pulses access ultrashort time spans to uncover microscopic details of dynamical processes. To date, however, it is technically challenging to produce two strong enough attosecond pulses for a pump-probe experiment, i.e., to start and stop the clock. One way to bypass this obstacle is the so-called streaking method [1]: An attosecond laser pulse starts the clock by emitting an electron. A second, weak near-infrared pulse, phase locked with a tunable delay $s$ to the attosecond pulse, "streaks" this photoelectron, i.e., influences its momentum in the continuum while it is leaving its (binding) potential. From the streaking spectrogram, the energy (or momentum) distribution as a function of $s$, one can extract so-called streaking delays $\tau_{s}$. These delays differ for photoelectrons coming from different orbitals, as revealed in seminal experiments for atoms and surfaces [2,3]. With photoelectron streaking having developed into a frequently used tool in attosecond physics, experiments have been extended to molecules, nanoparticles, and solids [4-8].

In the following, we will show that all photoionization time delays extracted by streaking-even relative ones between two orbitals with different binding energy - do not represent a property of the target only, but of the combined light-matter system. They grow with decreasing streaking frequency beyond all limits. This has considerable consequences for photoionization streaking time delays regarding their interpretation and general relevance. This result follows from analyzing the energy absorption of the

Published by the American Physical Society under the terms of the Creative Commons Attribution 4.0 International license. Further distribution of this work must maintain attribution to the author(s) and the published article's title, journal citation, and DOI. Open access publication funded by the Max Planck Society. released electron, the very process that underlies the streaking approach.

Furthermore, we derive analytically that only for a finiterange potential and in the limit of very low streaking frequency, streaking delays approach the Wigner-Smith (WS) time delay $\delta t$ from scattering theory $[9,10]$, revealing how much time a particle spends traversing a potential in comparison with a free particle with the same energy $E$. Previously, comparing theoretically obtained streaking delays and WS delays has provided evidence that both agree for short-range potentials and typical laser parameters [11]. Since those laser parameters fulfill the criterion of low frequency $(800 \mathrm{~nm}$ light corresponding to $1.5 \mathrm{eV}$ photon energy, which is small compared to typical electron excess energies), this finding for short-range potentials is consistent with our derivation.

However, finite streaking time delays for (long-range) Coulomb potentials as reported in the literature from streaking experiments and simulations, do not have a deeper meaning and are merely a consequence of the low but finite streaking frequency. From a broader perspective, this should have been expected as the WS time delay for Coulomb potentials is infinite, i.e., ill defined [10].

Basically all theoretical accounts of the streaking technique rely on the strong-field approximation (SFA), which neglects the potential for the continuum electron although the field-potential interaction is the source of energy absorption from the streaking laser (see various reviews [11-13], with the notable exception [14]). Moreover, streaking measurements are "classical clocks" [11]. Other than "quantum clocks" measuring time delays [15-17], they do not rely on quantum interferences. Classical streaking calculations have been shown to coincide with quantum calculations $[11,18]$, as long as there are no resonances in the continuum [17]. They are 
also closely related to wave packet formulations of the time delay [19]. Hence, we use classical dynamics in the following, which naturally allow us to include the potential and to work out the cornerstones of streaking time delays from energy absorption clearly.

The time delay of a "half-collision" [11] in the spherical potential $V(r)$ initiated at time $t=0$ with energy $E$ follows from the action difference [20]

$$
S(E)=\int_{0}^{\infty} d r\left[p(r)-p_{E}\right]
$$

through the energy derivative $\delta t=d S(E) / d E$, which yields

$$
\delta t=\int_{0}^{\infty} d r\left(\frac{1}{p(r)}-\frac{1}{p_{E}}\right)=\int_{0}^{\infty} d t\left(1-\frac{p(t)}{p_{E}}\right),
$$

where in the last expression $d r=p d t$ has been used. The time delay $\delta t$ measures how much more or less time it takes a particle to move in a potential $V$ with momentum $p(r)=$ $\sqrt{2[E-V(r)]}$ compared to a free particle with momentum $p_{E}=\sqrt{2 E}$. This is particularly obvious from the second equation in (2). As noted by Smith [10], for $\delta t$ to be well defined, the particle must be asymptotically free.

Figure 1 shows $\delta t$ for a short-range Yukawa potential (black line). The time delay is compared to streaking shifts $\tau_{s}$, with a laser field of frequency $\omega$ and peak vector potential $A=F / \omega$, where the field strength $F$ is so small such that $A \ll p_{E}$. (Below we use intensities such that $A=p_{E} / 100$.) The streaking field

$$
A_{s}(t)=-A \sin (\omega[t+s])
$$

can be shifted by a delay $s$ with respect to the particle's release at $t=0$, often achieved by attosecond photoionization [3-8,18,21-27]. In a full streaking scan, $s$ is varied

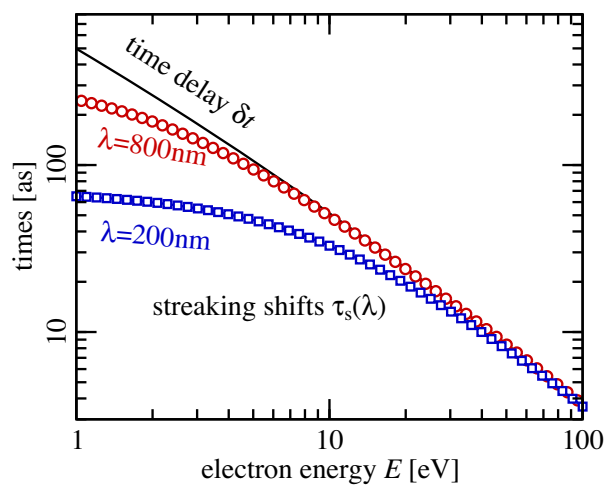

FIG. 1. Wigner-Smith time delay $\delta t$ (black solid line) and streaking delays $\tau_{s}$ for two wavelengths, $\lambda=200 \mathrm{~nm}$ (blue squares) and $\lambda=800 \mathrm{~nm}$ (red circles) as a function of the electron energy $E$ for a Yukawa potential $V(r)=e^{-r / r_{\text {yuk }}} / r$ with $r_{\text {yuk }}=3 \AA$. from $-T_{\omega} / 2$ to $+T_{\omega} / 2$, i.e., over the period $T_{\omega} \equiv 2 \pi / \omega$ of the streaking laser. Note that using a finite streaking pulse instead of (3) modifies the extracted $\tau_{s}$ only weakly. We show in Fig. 1 streaking shifts $\tau_{s}$ for two different laser wavelengths $\lambda$. They are determined from the shift relative to $A(t)$ of a sinusoidal function fitted to the calculated momentum change [cf. arrow in Fig. 2(b)] of the characteristic trajectory $r_{E}(t)$ starting at the potential center. Obviously, the time delay $\delta t$ agrees with those streaking shifts $\tau_{s}$ only for sufficiently large energies $E$.

In order to determine the general conditions under which streaking delays reveal the WS time delay, we investigate how the streaking field $A_{s}(t)$ changes the momentum of the electron while leaving the potential region. To first order in the vector potential $A$, the momentum change is given by

$\delta p(s)=A_{s}(0)+\frac{1}{p_{E}} \int_{0}^{\infty} d t\left[A_{s}(t)-A_{s}(0)\right] V^{\prime}\left(r_{E}(t)\right)$

with the vector potential $A_{s}(t)$ and all other quantities from the field-free $(A=0)$ dynamics. The derivation of Eq. (4) is straightforward in the Kramers-Henneberger frame and requires only the perturbative character of the streaking field $A \ll p_{E}$. Details can be found in the Supplemental Material [28].

We have written (4) in a form which makes the relation to the often-used SFA apparent. The latter neglects the potential $V$ leaving the well-known result that $\delta p(s)=A_{s}(0)$, the vector potential at release time $t=0$. We would like to stress at this point that all results below are obtained from exact numerical calculations, we use Eq. (4) based on the characteristic field-free trajectory $r_{E}(t)$ with energy $E$ for interpretation only. Figure 2 shows,

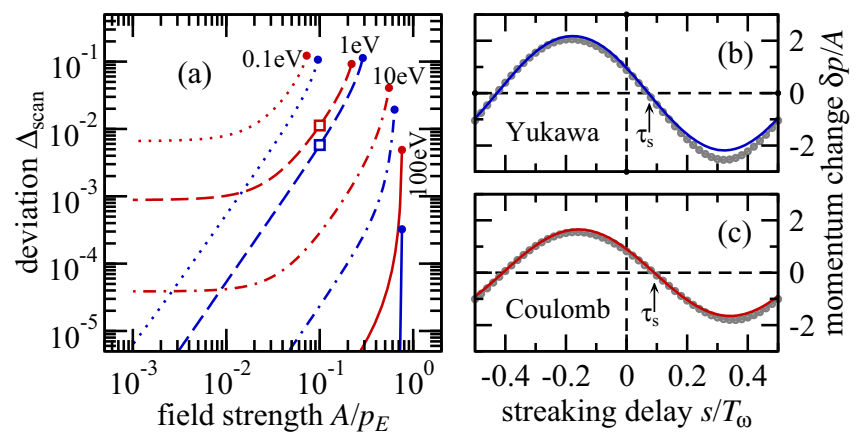

FIG. 2. Comparison of the harmonic streaking scans $\delta p(s)$ from Eq. (5) with respect to a numerical solution. The relative deviation is defined as $\Delta_{\text {scan }} \equiv \int d s[\delta p(s)-$ $\left.\delta p_{\text {num }}(s)\right]^{2} / \int d s\left[\delta p_{\text {num }}(s)\right]^{2}$ and shown for four energies (a) as a function of the laser field strength $A$, with $A$ given in units of $p_{E}$. Results are for the Yukawa potential, as in Fig. 1, (blue lines) and the Coulomb potential (red). The circles mark the largest $F$ for which a full streaking scan can be made. The squares mark the two examples, having relative large deviations $\Delta_{\text {scan }}$, shown to the right (b),(c), where $\delta p_{\text {num }}$ (gray circles) is compared to the harmonic expression $\delta p$ (blue and red lines) from Eq. (5). 
however, that it gives even quantitatively correct results for sufficiently small streaking fields $F$ or $A$, respectively.

Note that Eq. (4) is valid for arbitrary forms $A_{s}(t)$. For the harmonic streaking field (3) it has the particularly compact form

$$
\begin{aligned}
\delta p(s) & =-\frac{F}{\omega}\left[\chi_{S} \sin (\omega s)+\chi_{C} \cos (\omega s)\right], \\
\chi_{S} & \equiv 1-\frac{1}{p_{E}} \int_{0}^{\infty} d t[1-\cos (\omega t)] V^{\prime}\left(r_{E}(t)\right), \\
\chi_{C} & \equiv \frac{1}{p_{E}} \int_{0}^{\infty} d t \sin (\omega t) V^{\prime}\left(r_{E}(t)\right),
\end{aligned}
$$

which reveals that $\delta p$ is harmonic as well, with amplitude (in units of the quiver momentum $F / \omega$ ) and phase defined by the newly introduced quantities $\chi_{S}$ and $\chi_{C}$.

With the momentum change (5) we can elucidate the relation to the WS time delay (2), which can be rewritten (using integration by parts) as

$$
\delta t=-\frac{1}{p_{E}} \int_{0}^{\infty} d t t V^{\prime}\left(r_{E}(t)\right) .
$$

Without loss of generality we may compare (6) to the streaking-induced momentum change at $s=0$ from (5)

$$
\delta p(0)=-\frac{F}{\omega} \chi_{C}=-\frac{F}{p_{E}} \int_{0}^{\infty} d t \frac{\sin (\omega t)}{\omega} V^{\prime}\left(r_{E}(t)\right) .
$$

It is obvious that for small streaking frequencies $\omega, \delta p(0)$ directly gives the WS time delay

$$
\tau_{z} \equiv \frac{\delta p(0)}{F}=\frac{\chi_{C}}{\omega} \quad \text { with } \quad \delta t=\lim _{\omega \rightarrow 0} \tau_{z} .
$$

Since $\delta p$ is measurable, Eq. (8) provides a direct way to determine $\delta t$ experimentally, provided that the streaking frequency $\omega$ is sufficiently small and that the streaking vector potential $A$ is sufficiently weak to allow for a firstorder description of the momentum change or energy absorption, cf. Fig. 2. Note, that there is no need for a full streaking scan of many delays $s$, a single measurement with $s=0$ contains the necessary information.

Turning to traditional streaking measurements, the momentum change (5) may be written as

$$
\begin{aligned}
\delta p(s) & =-A \sqrt{\chi_{S}^{2}+\chi_{C}^{2}} \sin \left(\omega\left[s+\tau_{s}\right]\right), \\
\tau_{s} & \equiv \frac{1}{\omega} \arctan \left(\chi_{C}, \chi_{S}\right),
\end{aligned}
$$

which is, just as Eq. (5a), a harmonic function of $s$ with a displacement in the phase given by the streaking shift $\tau_{s}$. Both times $\tau_{z}$ and $\tau_{s}$ are, for sufficiently weak lasers, independent of the field strength $F$, as observed experimentally [3]. From $\lim _{\omega \rightarrow 0} \chi_{S}=1$ and $\lim _{\omega \rightarrow 0} \chi_{C}=\omega \delta t$, cf. Eq. (8), follows immediately

$$
\lim _{\omega \rightarrow 0} \tau_{s}=\lim _{\omega \rightarrow 0} \frac{\arctan (\omega \delta t, 1)}{\omega}=\delta t .
$$

Equations (8) and (10) are the main result of this work, since they show that both single and full-scan streaking measurements may indeed provide the Wigner-Smith time delay.

To be quantitative, we investigate next, under which conditions the streaking delays $\tau_{s}$ and $\tau_{z}$ really give the WS time delays $\delta t$ for a Yukawa potential, which has been used as prototypical short-range potential in this context [18]. Figure 3 provides the evolution of the relative differences $\Delta_{z} \equiv\left|\tau_{z}-\delta t\right| / \delta t$ and $\Delta_{s} \equiv\left|\tau_{s}-\delta t\right| / \delta t$ as a function of electron energy $E$ and streaking-laser wavelength $\lambda$. Indeed, for "typical" energies $E \gg 10 \mathrm{eV}$ and Ti:sapphire laser pulses $(\lambda=800 \mathrm{~nm})$ there is reasonable agreement between streaking shifts $\tau_{s}, \tau_{z}$, and time delays $\delta t$, as reported before $[11,18]$. However, this agreement is by no means generally guaranteed-it requires, depending on the energy $E$, sufficiently large wavelengths $\lambda$ in agreement with Eqs. (8) and (10). While the overall behavior of both streaking delays is similar, one can see that $\Delta_{s}$ grows faster than $\Delta_{z}$. Details regarding location and shape of the contour lines depend, of course, on the underlying potential. From Eqs. (5) follows

$$
r_{E}\left(T_{\omega} / 4\right) \gg r_{V},
$$

i.e., the trajectory with energy $E$ should have reached the asymptotic region beyond the potential's range $r_{V}$ after a quarter of the streaking-laser period $T_{\omega}$. Thus, larger energies and longer wavelengths (e.g., THz pulses [24])
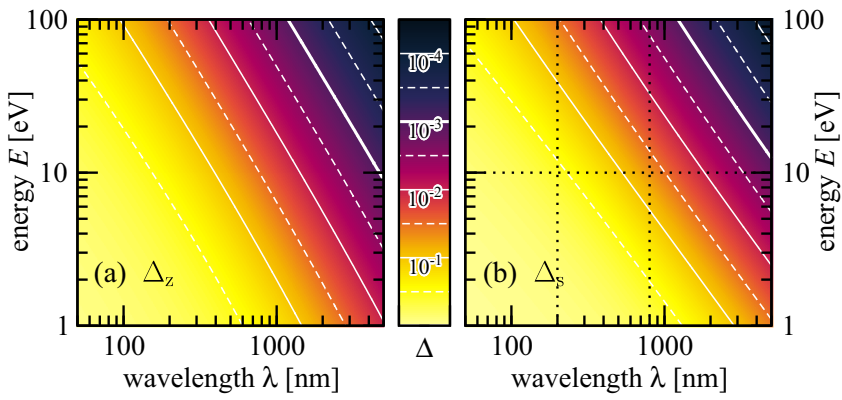

FIG. 3. Deviation of the zero-streaking time $\tau_{z}=\delta p(0) / F$ according to (8) and the full-streaking time $\tau_{s}$ according to (9) from the WS delay $\delta t$ for the Yukawa potential used before in Fig. 1. Shown are the relative errors $\Delta_{z} \equiv\left|\tau_{z}-\delta t\right| / \delta t$ and $\Delta_{s} \equiv$ $\left|\tau_{s}-\delta t\right| / \delta t$ as a function laser wavelength $\lambda$ and electron energy $E$. The vertical dotted lines mark the two wavelengths already shown in Fig. 1, the horizontal one the energy shown in Fig. 4(a). The thicker white lines are the lower bounds for $1 \%$ occuracy and can be approximately described by $E>135 \mathrm{eV}[\lambda / 1 \mu \mathrm{m}]^{-5 / 3}$ and $E>126 \mathrm{eV}[\lambda / 1 \mu \mathrm{m}]^{-3 / 2}$, respectively. 
are always an option for short-range potentials to reach the parameter regime where the streaking time delays approach $\delta t$.

This, however, does not hold for long-range potentials, most importantly, for the Coulomb potential. Since $\delta t$ diverges and the streaking delays $\tau_{z}$ and $\tau_{s}$ agree with $\delta t$ for $\omega \rightarrow 0$ or $\lambda \rightarrow \infty$, they should also diverge in this limit and in fact they do. Yet, the situation is more intricate as streaking for larger $\omega$ is only sensitive to a certain part of the potential (notably the inner part as shown below) and therefore leads to a finite momentum change. This is, however, not only a property of the Coulomb potential itself but depends on the parameters used, namely electron excess energy $E$ and streaking frequency $\omega$, as illustrated in Fig. 4. Note, that the relative streaking delay between photoelectrons from two orbitals with different binding energies, or equivalently, with two different excess energies, exhibits the same qualitative dependence on the streaking frequency [compare the black line in Fig. 4(b) with the red lines]. In other words, neither absolute streaking delays nor these relative streaking time delays, although finite, are suitable to characterize a Coulombic system.

From a theoretical point of view, one should take the pure (hydrogen's) Coulomb potential $V_{\text {ref }}(r)=-1 / r$ as a

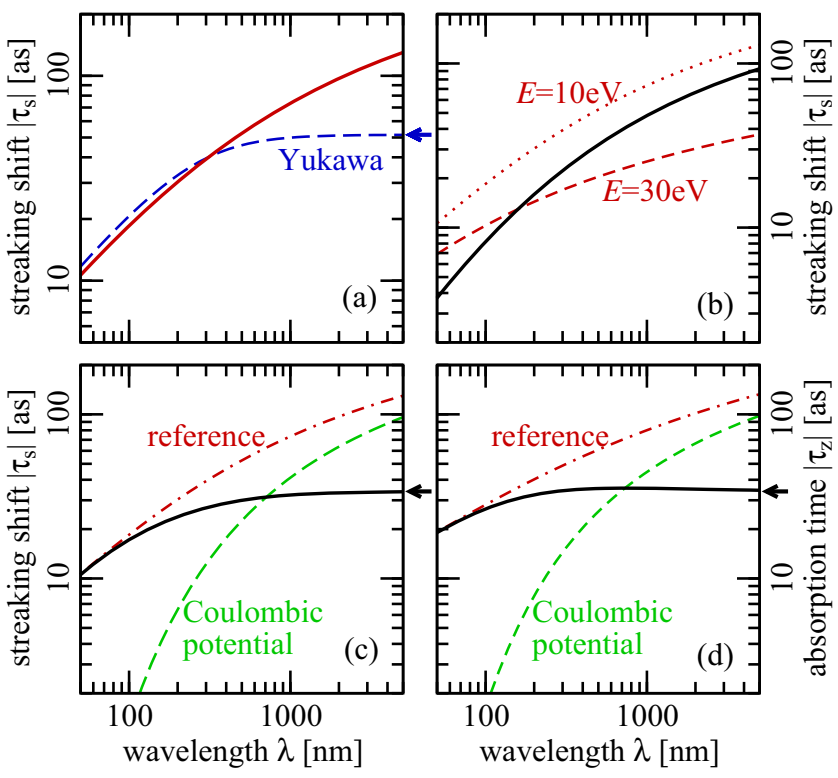

FIG. 4. Streaking shifts $\tau_{s}$ (and $\tau_{z}$ ) for Coulomb potentials as a function of the streaking-laser wavelength $\lambda$. In order to allow for a logarithmic scale we show absolute values for all times. (a) Comparison of Coulomb (red solid lines) and Yukawa, as in Fig. 1 (blue dashed) potential for $E=10 \mathrm{eV}$. (b) Shifts for two different energies (red dotted and red dashed) and their respective difference (black solid). (c),(d) $\tau_{s}$ and $\tau_{z}$ for the smoothed Coulomb potential $V(r)=-1 / \sqrt{r^{2}+r_{\mathrm{sm}}^{2}}$ with $r_{\mathrm{sm}}=3 \AA$ (green dashed) and in Eq. (13) defined $\tau_{\sigma}^{c}$ (black solid) using the Coulomb potential (red dot-dashed) as reference, both obtained for the same energy $E=10 \mathrm{eV}$. The arrows at the right give values for $\lambda=100 \mu \mathrm{m}$, indicating converged values. reference (instead of the free particle) to determine time delays of systems with a long-range Coulomb tail, an idea that has been put forward in the context of RABBIT [12]. That means we define a Coulomb action $S^{c}(E)$ by replacing in Eq. (1) the free-particle momentum $p_{E}$ by $p_{\text {ref }}(r)=$ $\sqrt{2\left[E-V_{\text {ref }}(r)\right]}$ or, equivalently, subtract the action of the hydrogen reference from the actual Coulomb-tailed system. We obtain a finite time delay

$$
\delta t^{c} \equiv \frac{d S^{c}(E)}{d E}=\frac{d S(E)}{d E}-\frac{d S_{\mathrm{ref}}(E)}{d E} .
$$

In analogy to (12) we define

$$
\tau_{\sigma}^{c} \equiv \tau_{\sigma}-\tau_{\sigma}^{\mathrm{ref}} \quad \text { with } \quad \sigma=s, z
$$

whereby the reference times obtained for $V_{\text {ref }}$ introduced in (12) above. Note that in Eq. (13) the individual terms diverge for $\omega \rightarrow 0$, the difference, however, has a finite limit, independent of the properties of the streaking field (if it is sufficiently weak), as illustrated with Figs. 4(c) and 4(d). This limit establishes the proper streaking time delays for potentials with a Coulomb tail.

We conclude with a remark on the so-called "Coulomblaser coupling time" $[11,18,27,29]$ — suggesting that in the case of a Coulomb potential there is a coupling between laser and potential and, conversely, that this is not the case for short-range potentials. In general, we have shown that streaking time delays originate from the momentum change $\delta p$ through the streaking laser. A momentum change of the electron is only possible in the presence of a potential as basic laws of energy and momentum conservation forbid energy absorption from light by a free electron [30]. We underline this point with Fig. 5, which shows the electron's energy as a function of distance $r$

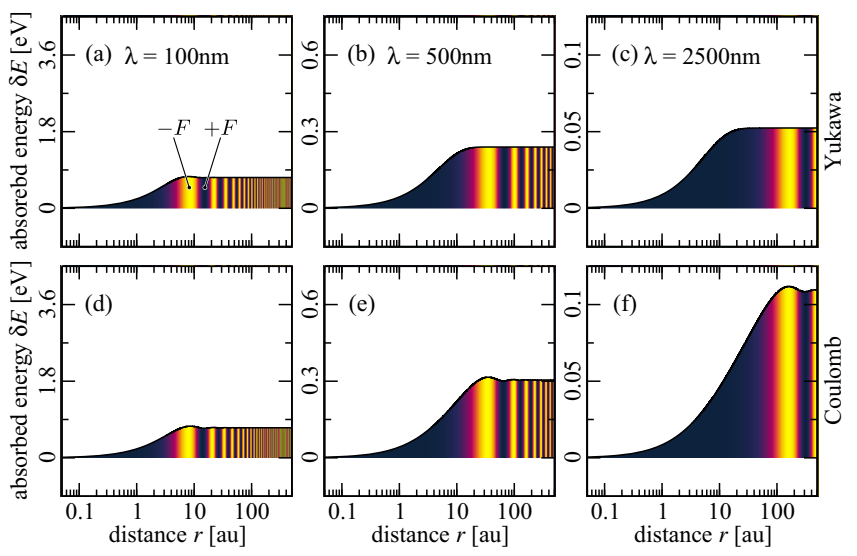

FIG. 5. Absorbed energy $\delta E(r)=E(r)-E$ as a function of distance $r$ for the Yukawa and the Coulomb potential for three different laser wavelengths $\lambda$, specified in the panels. The color coding represents the instantaneous laser field strength for the time when the electron is at a particular $r$. 


$$
E_{s=0}(r)=\int_{0}^{t(r)} d \tilde{t} A(\tilde{t}) V^{\prime}\left(r_{E}(\tilde{t})-[\alpha(\tilde{t})-\alpha(0)]\right),
$$

with the quiver motion $\alpha(t)$ in the Kramers-Henneberger picture. The index " $s=0$ " indicates that the electron is released at field maximum, vanishing vector potential or maximum excursion, respectively. As one can see energy absorption occurs early on, i.e., during the first half-cycle of the laser $t<T_{\omega} / 2$, cf. the respective leftmost dark stripes in Fig. 5, for both short- and long-range potentials. At the distance traveled in this time by the electron, the asymptotic values of energy absorption (or the asymptotic values of the integrals $\chi_{S}$ and $\chi_{C}$, respectively) are reached (apart from tiny, quickly vanishing oscillations) in accordance with Eq. (11) as can be seen in all panels of Fig. 5. Since for longer wavelengths $\lambda$ also $T_{\omega} / 2$ grows, the electron travels further outward (larger $r$ ) during this time span, giving rise to more energy absorption, unless this region is already beyond the range of the potential [which is the case for the Yukawa potential in Fig. 5(c)]. Since the Coulomb potential has an infinite range, energy absorption continues to grow with increasing laser period (smaller frequency) as seen in Figs. 5(d)-5(f).

In summary, we have derived the streaking time delays by means of the physically underlying process of energy absorption from the streaking laser which determines the measurable momentum of the photoelectron. Our analysis has shown that streaking time delays become independent of the properties of the streaking laser and approach the Wigner-Smith time delay of short-range potentials for sufficiently weak streaking fields and in the limit of small frequencies or long wavelengths. That streaking experiments for atoms measure finite times $\tau_{s}$ (despite infinite WS time delays $\delta t$ for Coulombic systems) is a consequence of finite laser frequencies used. Those finite streaking delays, however, depend on the laser parameters and the excess energy of the electron. Therefore, both absolute streaking delays and relative ones for different excess energies are not suitable to characterize properties of a Coulombic system. From a theoretical point of view, photoionization streaking delays should be measured relative to those of a pure hydrogenic Coulomb potential at the same excess energy of the photoelectron. Since this is impossible with a single experiment, it is probably more feasible experimentally to use a reference system with an orbital binding energy as close as possible to the one to be investigated, as recently done in a different context [31].

Obviously, the interpretation of measured streaking time delays requires careful analysis. Even a relative measurement of a time delay between two orbitals of different binding energy in the same atom and with the same streaking laser depends against any reasonable expectation on the properties of the streaking light, namely its frequency. However, with the results presented here, it should be possible in the future to design experiments which measure time delays free from properties of the streaking laser.

[1] E. Constant, V. D. Taranukhin, A. Stolow, and P. B. Corkum, Methods for the measurement of the duration of high-harmonic pulses, Phys. Rev. A 56, 3870 (1997).

[2] A. L. Cavalieri, N. Müller, T. Uphues, V. S. Yakovlev, A. Baltuška, B. Horvath, B. Schmidt, L. Blümel, R. Holzwarth, S. Hendel, M. Drescher, U. Kleineberg, P. M. Echenique, R. Kienberger, F. Krausz, and U. Heinzmann, Attosecond spectroscopy in condensed matter, Nature (London) 449, 1029 (2007).

[3] M. Schultze, M. Fieß, N. Karpowicz, J. Gagnon, M. Korbman, M. Hofstetter, S. Neppl, A. L. Cavalieri, Y. Komninos, T. Mercouris, C. A. Nicolaides, R. Pazourek, S. Nagele, J. Feist, J. Burgdörfer, A. M. Azzeer, R. Ernstorfer, R. Kienberger, U. Kleineberg, E. Goulielmakis, F. Krausz, and V.S. Yakovlev, Delay in photo-emission, Science 328, 1658 (2010).

[4] S. Neppl, R. Ernstorfer, A. L. Cavalieri, C. Lemell, G. Wachter, E. Magerl, E. M. Bothschafter, M. Jobst, M. Hofstetter, U. Kleineberg, J. V. Barth, D. Menzel, J. Burgdörfer, P. Feulner, F. Krausz, and R. Kienberger, Direct observation of electron propagation and dielectric screening on the atomic length scale, Nature (London) 517, 342 (2015).

[5] M. Huppert, I. Jordan, D. Baykusheva, A. von Conta, and H. J. Wörner, Attosecond Delays in Molecular Photoionization, Phys. Rev. Lett. 117, 093001 (2016).

[6] F. Siek, S. Neb, P. Bartz, M. Hensen, C. Strüber, S. Fiechter, M. Torrent-Sucarrat, V. M. Silkin, E. E. Krasovskii, N. M. Kabachnik, S. Fritzsche, R. D. Muiño, P. M. Echenique, A. K. Kazansky, N. Müller, W. Pfeiffer, and U. Heinzmann, Angular momentum-induced delays in solid-state photoemission enhanced by intra-atomic interactions, Science 357, 1274 (2017).

[7] L. Seiffert, Q. Liu, S. Zherebtsov, A. Trabattoni, P. Rupp, M. C. Castrovilli, M. Galli, F. Süßmann, K. Wintersperger, J. Stierle, G. Sansone, L. Poletto, F. Frassetto, I. Halfpap, V. Mondes, C. Graf, E. Rühl, F. Krausz, M. Nisoli, T. Fennel, F. Calegari, and M. F. Kling, Attosecond chronoscopy of electron scattering in dielectric nanoparticles, Nat. Phys. 13, 766 (2017).

[8] M. Ossiander, J. Riemensberger, S. Neppl, M. Mittermair, M. Schäffer, A. Duensing, M. S. Wagner, R. Heider, M. Wurzer, M. Gerl, M. Schnitzenbaumer, J. V. Barth, F. Libisch, C. Lemell, J. Burgdörfer, P. Feulner, and R. Kienberger, Absolute timing of the photo-electric effect, Nature (London) 561, 374 (2018).

[9] E. P. Wigner, Lower limit for the energy derivative of the scattering phase shift, Phys. Rev. 98, 145 (1955).

[10] F. T. Smith, Lifetime matrix in collision theory, Phys. Rev. 118, 349 (1960).

[11] R. Pazourek, S. Nagele, and J. Burgdörfer, Attosecond chronoscopy of photo-emission, Rev. Mod. Phys. 87, 765 (2015).

[12] J. M. Dahlström, A. L'Huillier, and A. Maquet, Introduction to attosecond delays in photo-ionization, J. Phys. B 45, 183001 (2012). 
[13] A. Maquet, J. Caillat, and R. Taïeb, Attosecond delays in photo-ionization: Time and quantum mechanics, J. Phys. B 47, 204004 (2014).

[14] C.-H. Zhang and U. Thumm, Electron-ion interaction effects in attosecond time-resolved photoelectron spectra, Phys. Rev. A 82, 043405 (2010).

[15] K. Klünder, J. M. Dahlström, M. Gisselbrecht, T. Fordell, M. Swoboda, D. Guénot, P. Johnsson, J. Caillat, J. Mauritsson, A. Maquet, R. Taïeb, and A. L'Huillier, Probing Single-Photon Ionization on the Attosecond Time Scale, Phys. Rev. Lett. 106, 143002 (2011).

[16] J. M. Dahlström, D. Guénot, K. Klünder, M. Gisselbrecht, J. Mauritsson, A. L'Huillier, A. Maquet, and R. Taïeb, Theory of attosecond delays in laser-assisted photo-ionization, Chem. Phys. 414, 53 (2013).

[17] M. Isinger, R. J. Squibb, D. Busto, S. Zhong, A. Harth, D. Kroon, S. Nandi, C. L. Arnold, M. Miranda, J. M. Dahlström, E. Lindroth, R. Feifel, M. Gisselbrecht, and A. L'Huillier, Photo-ionization in the time and frequency domain, Science 358, 893 (2017).

[18] R. Pazourek, S. Nagele, and J. Burgdörfer, Time-resolved photo-emission on the attosecond scale: Opportunities and challenges, Faraday Discuss. 163, 353 (2013).

[19] U. Thumm, Q. Liao, E. M. Bothschafter, F. Süßmann, M. F. Kling, and R. Kienberger, Attosecond physics: Attosecond streaking spectroscopy of atoms and solids, in Fundamentals of Photonics and Physics Vol. 1, edited by D. L. Andrews (John Wiley \& Sons, Hoboken, 2015).

[20] J. M. Rost, Semiclassical $S$-matrix theory for atomic fragmentation, Phys. Rep. 297, 271 (1998).

[21] R. Kienberger, E. Goulielmakis, M. Uiberacker, A. Baltuška, V. Yakovlev, F. Bammer, A. Scrinzi, T. Westerwalbesloh, U. Kleineberg, U. Heinzmann, M. Drescher, and F. Krausz, Atomic transient recorder, Nature (London) 427, 817 (2004).
[22] J. Itatani, F. Quéré, G. L. Yudin, M. Y. Ivanov, F. Krausz, and P. B. Corkum, Attosecond Streak Camera, Phys. Rev. Lett. 88, 173903 (2002).

[23] V.S. Yakovlev, F. Bammer, and A. Scrinzi, Attosecond streaking measurements, J. Mod. Opt. 52, 395 (2005).

[24] U. Frühling, M. Wieland, M. Gensch, T. Gebert, B. Schütte, M. Krikunova, R. Kalms, F. Budzyn, O. Grimm, J. Rossbach, E. Plönjes, and M. Drescher, Single-shot terahertz-field-driven x-ray streak camera, Nat. Photonics 3, 523 (2009).

[25] A. S. Kheifets and I. A. Ivanov, Delay in Atomic PhotoIonization, Phys. Rev. Lett. 105, 233002 (2010).

[26] C.-H. Zhang and U. Thumm, Streaking and Wigner time delays in photo-emission from atoms and surfaces, Phys. Rev. A 84, 033401 (2011).

[27] M. Ivanov and O. Smirnova, How Accurate is the Attosecond Streak Camera?, Phys. Rev. Lett. 107, 213605 (2011).

[28] See Supplemental Material at http://link.aps.org/ supplemental/10.1103/PhysRevLett.125.113202 for deriving the electron's momentum change (corresponding to energy absorption) in detail.

[29] G. Schmid, K. Schnorr, S. Augustin, S. Meister, H. Lindenblatt, F. Trost, Y. Liu, N. Stojanovic, A. AlShemmary, T. Golz, R. Treusch, M. Gensch, M. Kübel, L. Foucar, A. Rudenko, J. Ullrich, C. D. Schröter, T. Pfeifer, and R. Moshammer, Terahertz-Field-Induced Time Shifts in Atomic Photo-Emission, Phys. Rev. Lett. 122, 073001 (2019).

[30] J. M. Rost, Analytical total photo cross section for atoms, J. Phys. B 28, L601 (1995).

[31] N. Camus, E. Yakaboylu, L. Fechner, M. Klaiber, M. Laux, Y. Mi, K. Z. Hatsagortsyan, T. Pfeifer, C. H. Keitel, and R. Moshammer, Experimental Evidence for Quantum Tunneling Time, Phys. Rev. Lett. 119, 023201 (2017). 